\title{
NEAR-INFRARED SPECTROSCOPY OF INTERSTELLAR DUST
}

\author{
F. Boulanger ${ }^{1}$, T. Onaka ${ }^{2}$, P. Pilleri ${ }^{3,4}$ and C. Joblin ${ }^{3,4}$
}

\begin{abstract}
Near infrared observations of reflection nebulae have set the historical ground for the discovery of interstellar PAHs, but since, space observations have focused on their mid-IR features, and data shortward of $5 \mu \mathrm{m}$ have remained scarce. The Spitzer/IRAC images in the 3.6 and $4.5 \mu \mathrm{m}$ channels do show that the near-IR emission from small dust particles is ubiquitous across the Galaxy, but provide no spectroscopic information. To investigate the nature of this near-IR dust emission, we have obtained AKARI spectroscopic observations, over the $2.5-5 \mu \mathrm{m}$ spectral range, for a set of archetype PDRs mapped with the Spitzer spectrometer at mid-IR wavelengths. These AKARI data supplement earlier observations with the SWS ISO spectrometer, in providing the gain in sensitivity needed to observe low excitation sources, and the spatial information required to spatially correlate near-IR spectroscopic signatures with physical conditions and observed changes in mid-IR spectra. This paper presents the first results of the data analysis, in relation to two open questions on interstellar PAHs. (1) Is there an evolutionary link from aliphatic carbon dust to PAHs? (2) What is the origin of the near-IR dust continuum? The AKARI spectra display features longward of the main $3.29 \mu \mathrm{m}$ PAH feature, and continuum emission. The intensity ratio between the features ascribed to aliphatic $\mathrm{CH}$ bonds and the $3.29 \mu \mathrm{m}$ aromatic band, varies spatially in a way that may be interpreted as evidence for aromatization of the smallest dust particles by photo-processing. The continuum displays a striking stepincrease across the $3.29 \mu \mathrm{m}$ feature. We also present a spectrum of a photodissociation region with a feature at $4.65 \mu \mathrm{m}$, which has been speculated to be related to the CD stretch in aliphatic hydrocarbon side-groups on PAHs.
\end{abstract}

\footnotetext{
${ }^{1}$ Institut d'Astrophysique Spatiale (IAS), UMR 8617, CNRS \& Université Paris-Sud 11, Bâtiment 121, 91405 Orsay Cedex, France

2 Department of Astronomy, University of Tokyo, Tokyo 113-0033, Japan

${ }^{3}$ Université de Toulouse, UPS, CESR, 9 avenue du colonel Roche, 31028 Toulouse, France

4 CNRS, UMR 5187, 31028 Toulouse, France
} 


\section{Introduction}

The discovery of near infrared (Near-IR) extended emission in reflection nebulae (Sellgren et al. 1983) initiated a new field of observations and research on interstellar dust. To account for the $3.3 \mu \mathrm{m}$ feature and the associated mid-IR features Léger \& Puget (1984) proposed that the emission follows stochastic heating of polycyclic aromatic hydrocarbon molecules (PAHs). The PAH emission features have since been observed to be a ubiquitous spectroscopic signature of dust in galaxies. Very small carbon dust particles (PAH molecules and very small grains) are established as a major constituent of interstellar dust that play key roles in the physics and chemistry of the interstellar medium (ISM), but discussions at this conference have shown that the origin and nature of these particles are still debated.

Near-IR wavelengths are of particular relevance to characterize the smallest particles of interstellar dust. First, the near-IR dust emission is thought to arise from the very smallest particles with less than $\sim 100$ carbon atoms. Second, emission in the $\mathrm{C}-\mathrm{H}$ stretching modes in the 3.3 to $3.5 \mu \mathrm{m}$ range are best suited to probe the presence of aliphatic side groups to PAHs. Other characteristic modes of $\mathrm{CH}_{3}$ and $\mathrm{CH}_{2}$ bonds that fall at $\sim 6.9$ and $\sim 7.2 \mu \mathrm{m}$ are more difficult to observe due to the presence of the 6.2 and $7.7 \mu \mathrm{m}$ PAH emission band. Third, one may probe the physics of the particle emission by characterizing the emission shortward and longward of the $\mathrm{C}-\mathrm{H}$ stretch bands.

Despite its specific interest, the near-IR spectral range has been barely explored (Geballe et al. 1989). The IRAS, IRTS, ISO and Spitzer space missions have provided us with a wealth of mid-IR observations, but spectroscopy shortward of $5 \mu \mathrm{m}$ is only available for bright sources with a high UV field (Verstraete et al. 2001; van Diedenhoven et al. 2004), such as the Orion Bar and the M17SW photodissociation regions (PDRs), and with little spatial information. The AKARI warm mission has provided the opportunity to obtain near-IR spectroscopy of diffuse emission with unprecedented sensitivity (Ohyama et al. 2007). In this paper, we briefly present our AKARI observing program, and the first results of the data analysis. This is an early account of on-going work, which will lead to a more thorough publication in a refereed journal.

\section{AKARI observations}

Our AKARI observing program was designed to investigate dust emission in PDRs. We used the AKARI grating spectrometer with an entrance slit 50" long. Our targets comprise 15 slit positions selected within six archetype star forming regions, on the basis of ISO and Spitzer mid-IR spectro-imaging observations. The list of targets includes objects with different excitation conditions (UV radiation field from 100 to a few 1000 times the Solar Neighborhood value), which show strong variation in their mid-IR spectra (C-C to $\mathrm{C}-\mathrm{H}$ features intensity ratio and band/continuum contrast). It also spans a range of distances, from the local ISM to the 30 Doradus LMC star forming region, to explore a range of physical scales. 

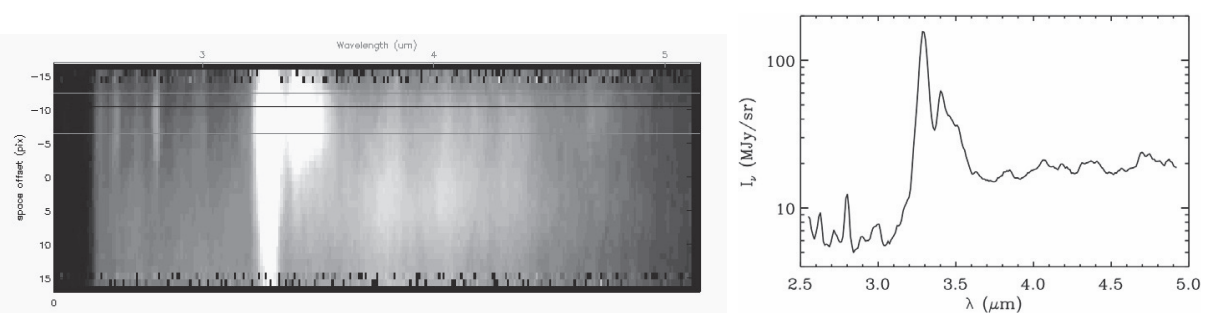

Fig. 1. AKARI observation of one pointing towards the reflection nebula NGC 7023. The spectral image is displayed to the left (wavelengths increasing from the left to the right). To the right, we show the spectrum extracted by averaging data within the two red lines at the top of the image. The log-scale highlights the fainter features at longer wavelengths, and the near-IR continuum with its step-increase across the $3.3-3.5 \mu \mathrm{m}$ range.

These AKARI data offer the combination of spatial (along the slit) and spectral information, required to spatially correlate near-IR spectroscopic signatures with physical conditions and mid-IR spectro-imaging observations.

The data have been reduced and calibrated by the Japanese team members using the best knowledge of the instrument (see Onaka et al. elsewhere in this volume). The outcome of the data reduction is a spectral image from which spectra can be extracted as a function of position along the slit (Fig. 1). The spectral resolution, measured on hydrogen recombination lines, ranges from 90 to 160 . We use an observation of HII gas emission towards 30 Doradus to validate the spectrometer capability to perform photometry of extended emission. This HII spectrum shows 10 hydrogen lines and continuum emission (free-free + free-bound), which are both in agreement with predictions from photo-ionization models. Spectral lines with intensities down to $2-3 \mathrm{MJy} / \mathrm{sr}$, and a $5 \mathrm{MJy} / \mathrm{sr}$ continuum, are reliably measured.

\section{AKARI dust spectra}

To illustrate the initial results of the data analysis, we present spectra measured for one slit position in the reflection nebula NGC 7023 (Figs. 1 and 2), and a spectrum of a molecular cloud in the Large Magellanic Cloud (Fig. 3). This last spectrum obtained towards the massive star forming region 30 Doradus includes several hydrogen recombination lines. In Figure 3, the gas emission, lines and continuum, has been removed using a photo-ionization model. The near-IR emission from dust comprises the prominent $3.29 \mu \mathrm{m}$ feature, a weaker spectrally structured plateau extending to $3.6 \mu \mathrm{m}$, and continuum emission. The step increase of the continuum emission from short to long wavelengths, across the $3.3-3.5 \mu \mathrm{m}$ range, is a remarkable result, which is observed in all spectra.

The $3.29 \mu \mathrm{m}$ feature is assigned to the $\mathrm{v}=1-0$ aromatic $\mathrm{C}-\mathrm{H}$ stretch transition. Emission in this feature is predicted to come mainly from neutral PAHs (DeFrees et al. 1993). Several types of transitions, most likely the $\mathrm{C}-\mathrm{H}$ stretch 

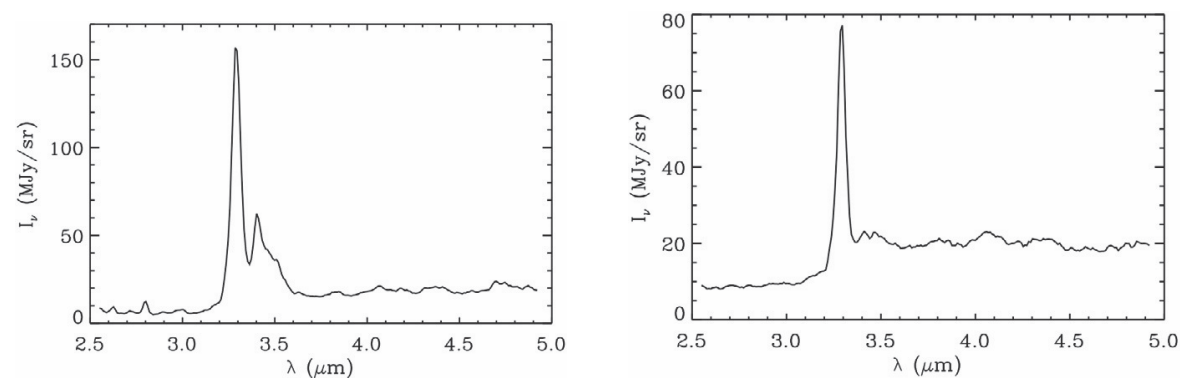

Fig. 2. AKARI spectra at two positions within the reflection nebula NGC 7023. The two positions sample the variation in intensity ratios of the mid-IR PAH features, which has been interpreted as evidence of the transition from neutral to cation PAHs (Rapacioli et al. 2005; Berné et al. 2007). From the left to the right spectra, the AKARI observations show the disappearance of the $3.4 \mu \mathrm{m}$ emission feature, and an increase by a factor 2 of the intensity of the continuum relative to that of the $3.3 \mu \mathrm{m}$ emission feature.

in aliphatic side groups attached to PAHs or in sur-hydrogenated PAHs, but also possibly, combination bands and hot bands including the $\mathrm{v}=2-1$ aromatic $\mathrm{C}-\mathrm{H}$ transition, also contribute to the plateau and superimposed features (Allamandola et al. 1989; Joblin et al. 1996; Bernstein et al. 1996). The near-IR continuum has been observed to be a dominant component of the near-IR emission from the diffuse ISM (Flagey et al. 2006), but it has never received a definitive interpretation within the PAH hypothesis. The step-increase in the continuum intensity across the $3.29 \mu \mathrm{m}$ (Fig. 1) band strongly suggests that the continuum is associated with the de-excitation of PAHs, but the emission process has still to be characterized. Allamandola et al. (1989) proposed that the continuum represents emission though vibrational modes, which become weakly active in excited PAHs. The combination of a large number of weak overlapping bands would make a quasi-continuum with little spectral structure. Within this interpretation, the step increase of the continuum emission across the $\mathrm{C}-\mathrm{H} 3.29 \mu \mathrm{m}$ feature could reflect the lower coupling efficiency between $\mathrm{C}-\mathrm{C}$ and $\mathrm{C}-\mathrm{H}$ modes than between $\mathrm{C}-\mathrm{C}$ modes. Fluorescence emission from an electronic excited state provides an alternative interpretation (Léger et al. 1988). Modelling (private communication from Pino and Schmidt) supported by experimental data suggests that, in PAH cations, the energy gap between the first excited state and the ground state may be small enough for cations to fluoresce in the near-IR. A more detailed analysis of the data will allow us to test this interpretation by looking for a spatial correlation between the continuum emission and that of PAH cations inferred from the decomposition of mid-IR spectra (Rapacioli et al. 2005; Berné et al. 2007).

\section{Evidence for small dust processing}

The nature of the small carbon dust particles has to be addressed within the broad context of the evolutionary cycle that can link them to the amorphous 

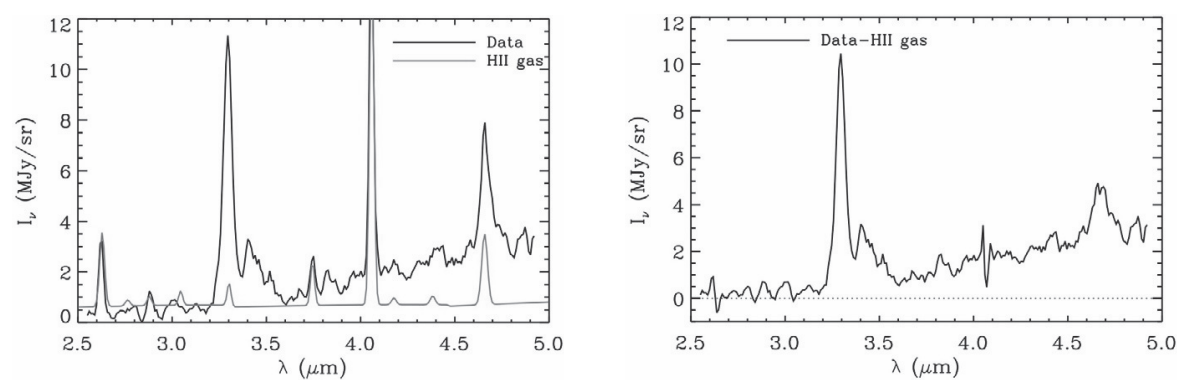

Fig. 3. AKARI spectrum of a molecular cloud in the Large Magellanic Cloud. The plot shows the emission spectrum with the dust and hydrogen lines to the left, and the spectrum obtained after subtraction of the gas emission to the right. We are confident that the $4.65 \mu \mathrm{m}$ feature is real. We have no interpretation for this band at present.

hydrogenated carbon dust seen in absorption spectroscopy (Dartois in this volume). Interstellar PAHs and VSGs could be produced by photo and/or mechanical fragmentation in grain-grain collisions involving amorphous carbon grains (Jones et al. 1996) or PAH clusters (Rapacioli et al. 2006). The free fragments are expected to be progressively photo-processed by selective dissociation of side groups and loss of non-aromatic $\mathrm{CH}$ bonds, when they are exposed to increasing ultraviolet photon fluxes as they move out of the shielded interiors of molecular clouds. This evolutionary scenario can be tested by studying the evolution of the $3.4 \mu \mathrm{m}$ aliphatic band relative to the $3.3 \mu \mathrm{m}$ aromatic band. In particular, we plan to study whether these variations are spatially correlated with variations in emission components inferred from mid-IR spectro-imaging observations (Rapacioli et al. 2005; Berné et al. 2007).

Onaka et al. (elsewhere in this volume) report large variations of the emission ratio, $3.4+3.5 \mu \mathrm{m}$ to $3.3 \mu \mathrm{m}$, for the diffuse Galactic emission. We observe similar variations on smaller scales within nearby PDRs. These results extend groundbased observations on brighter sources (Geballe et al. 1989; Joblin et al. 1996). The two spectra of NGC 7023 in Figure 2 are taken at the opposite ends of the $50^{\prime \prime}$ long slit, within and out of the dense surface of the molecular cloud (left and right spectra, respectively), marked by bright filamentary structure in PAH and near-IR $\mathrm{H}_{2}$ images. The disappearance of the $3.4 \mu \mathrm{m}$ emission feature may be interpreted as evidence for aromatization of the smallest dust particles by photo-processing. A thorough analysis of the spatial variations of the aliphatic to aromatic emission ratio across the NGC $7023 \mathrm{PDR}$ is required to ascertain and fully quantify this interpretation.

\section{Looking for new spectral features}

The AKARI spectra offer an unprecedented opportunity to look for faint near-IR spectral features. The C-D stretching mode around $4.5 \mu \mathrm{m}(4.4$ and $4.65 \mu \mathrm{m}$ for 
the aromatic/aliphatic C-D bond, respectively) is of particular interest to test if PAHs are highly deuterated (Draine 2004; Peeters et al. 2004). The C-D inplane and out-of-plane bending modes, expected at 11.7 and $15.4 \mu \mathrm{m}$, cannot be unambiguously identified, because they fall in a spectral region where other PAH features are present.

Analyses of spectra obtained with the Far Ultraviolet Spectroscopic Explorer (FUSE) satellite, together with spectra from the Copernicus space mission and ISM absorption probe spectrograph (IMAPS), have revealed a factor 4 variation in the observed D/H ratios for interstellar gas in the Solar Neighborhood (Linsky et al. 2006). Linsky et al. argue that spatial variations in the depletion of deuterium onto dust grains can explain the variations in the observed gas-phase $\mathrm{D} / \mathrm{H}$ ratios. This interpretation is supported by the correlation of $\mathrm{D} / \mathrm{H}$ with the gas column density and depletions of the refractory metals iron and silicon. Gas-grain reactions could lead to a high deuteration of hydrogenated carbonaceous grains and PAHs (Draine 2004). The gain in sensitivity provided by AKARI permit to look for deuterated PAHs in sources, less excited than the Orion Bar and M17SW PDRs studied in Peeters et al. (2004), which are more directly relevant to the deuteration of PAHs in the diffuse ISM (Onaka et al. in this volume).

We are presently cautious in reporting new spectral features, but we are not aware of any data artifact that could contaminate the emission feature at $4.65 \mu \mathrm{m}$ in Figure 3. We are thus confident that this feature is real. Its central wavelength matches that of the aliphatic C-D stretch mode (Peeters et al. 2004). However, the absence of the corresponding aromatic band at $4.4 \mu \mathrm{m}$, and the intensity of the feature - comparable to that of the $3.4 \mu \mathrm{m}$ - make the identification with deuterated aliphatic side groups on PAHs unsure.

We thank several participants of the conference, in particular Lou Allamandola, Philippe Bréchignac, Thomas Pino, Tim Schmidt, Kris Sellgren, and Xander Tielens, for discussions that helped us with the interpretation of the data.

\section{References}

Allamandola, L.J., Tielens, A., \& Barker, J.R., 1989, ApJS, 71, 733

Berné O., Joblin, C., Deville, Y., et al., 2007, A\&A, 469, 575

Bernstein, M.P., Sandford, S.A., \& Allamandola, L.J., 1996, ApJ, 472, L127

DeFrees, D.J., Miller, M.D., Talbi, D., et al., 1993, ApJ, 408, 530

Draine, B.T., 2004, in Astrophysics in the Far Ultraviolet [Astro-ph/0410310]

Draine, B.T., \& Li, A., 2007, ApJ, 657, 810

Flagey N., Boulanger F., Verstraete, L., et al., 2006, A\&A, 453, 969

Geballe, T.R., Tielens, A.G.G.M., Allamandola, L.J., et al., 1989, ApJ, 341, 278

Joblin, C., Tielens, A.G.G.M., Allamandola, L.J., \& Geballe, T.R., 1996, ApJ, 458, 610

Jones, A.P., Tielens, A.G.G.M., \& Hollenbach, D.J., 1996, ApJ, 469, 740

Léger, A., \& Puget, J.L., 1984, A\&A, 137, L5

Léger, dHendecourt, L., \& Boissel, P., 1988, PhRvL, 60, 921L 
Linsky, J., Draine, B.T., Moos, H.W., et al., 2006, ApJ, 647, 1106 Ohyama, Y., Onaka, T., Matsuhara, H., et al., 2007, PASJ, 59, S411 Peeters, E., Allamandola, L.J., Bauschlicher, C.W., et al., 2004, ApJ, 604, 252 Rapacioli, M., Joblin, C., \& Boissel, P., 2005, A\&A, 429, 193

Rapacioli, M., Calvo, F., Joblin, C., et al., 2006, A\&A, 460, 519

Sellgren, K., Werner, M.W., \& Dinerstein, H.L., 1983, ApJ, 271, L13

van Diedenhoven, B., Peeters, E., Van Kerckhoven, C., et al., 2004, ApJ, 611, 928

Verstraete, L., Pech, C., Moutou, C., et al., 2001, A\&A, 372, 981 
